\title{
PROPUESTAS METODOLÓGICAS PARA LA EDUCACIÓN EN DERECHOS HUMANOS EN LOS TEXTOS DE LAS NACIONES UNIDAS Y LA UNESCO
}

Carolina Ugarte y Concepción Naval

\section{RESUMEN}

La educación en derechos humanos es un medio idóneo para contribuir al desarrollo pleno de la personalidad. Aunque no puede hablarse de personas educadas en los derechos humanos hasta que éstos sean vida en la conducta personal, es cierto que su realización también depende de la existencia de un clima político e institucional favorable al respeto y promoción de tales derechos. A este respecto, las Naciones Unidas desempeñan un papel relevante, ya que velan por la promoción y protección de lo humano en el mundo. En este sentido, partiendo de los textos de las Naciones Unidas y la UNESCO -que recogen la cuestión de la educación en derechos humanos-, se delinearán las propuestas metodológicas de estos organismos con relación a este aspecto educativo. Las líneas apuntadas en los textos de organismos internacionales, se completan añadiendo algunos comentarios ocasionales.

\section{ABSTRACT}

Human Rights Education is an efficient way to contribute to the integral development of the personality. Though we cannot speak about buman rights educated people until these rights are alive in personal behaviour, it is true that their 
realization depends of a favourable institutional and political atmosphere of respect and promotion of human rights. In this way, the United Nations have an important role, because they look after the promotion and protection of the human condition in the world. Then, we analyse the texts of the United Nations and the UNESCO that includes the topic of human rights education. We try to know the methodological proposals of these organisms in relation to this educational aspect. The international organizations guidelines are complemented with some comments.

Palabras clave: Educación; derechos humanos; UNESCO; ONU; metodologías.

\section{INTRODUCCIÓN}

Los textos internacionales de derechos humanos son un instrumento creado para reconocer, proteger y promover el respeto y cumplimiento universal de estos derechos. La "Declaración" y todo el sistema internacional de protección de los derechos humanos son referentes útiles de los cuales partir para después intentar promover más eficazmente su defensa, respeto y ejercicio y, en concreto, el derecho a la educación ${ }^{1}$.

Cfr. Álvarez Vita, J. (1998)., "De la Declaración Universal de Derechos Humanos a la globalización: medio siglo de camino (1948-1998)", en Política Internacional., octubre-diciembre (1998)., pp.53-81; Martínez, M. y Noguera, E. (1998)., "La Declaración Universal de Derechos Humanos: compromisos y deberes", en Revista Española de Pedagogía., 211., pp.483-510; Medina, R. (2002)., La formación en los valores de los derechos humanos, fundamento de la convivencia y la paz., Madrid., Real Academia de Doctores; y del mismo autor: "Los derechos humanos y la educación en los valores de una ciudadanía universal", en Revista Española de Pedagogía., 211., pp.529-559. 
En la "Declaración Universal de Derechos Humanos" de 1948 se reconoce el derecho a la educación como derecho humano fundamental: "Toda persona tiene derecho a la educación" ${ }^{2}$ (artículo 26.1). Y precisamente, uno de sus componentes es la educación en derechos humanos. Ésta es educación en sentido pleno, ya que ayuda a alcanzar el desarrollo personal en plenitud, fin primordial al que se orienta la educación. En este sentido, la educación en derechos humanos destaca el núcleo de la auténtica educación y, por ello, forma parte del derecho a la educación ${ }^{3}$.

De una reflexión sobre el contenido de la educación en derechos humanos, puede afirmarse que ésta es un medio idóneo para afirmar la dignidad humana, contribuir al desarrollo personal pleno, fomentar el respeto a los demás derechos humanos, estimular la participación social y favorecer el respeto a uno mismo y a los demás ${ }^{4}$.

En esta línea, podríamos afirmar que la cuestión de la educación en derechos humanos goza de plena vigencia ${ }^{5}$.

\section{Artículo 26.1.}

3 Cfr. Medina, R., "Educación moral y comportamiento cívico-político", en Revista Española de Pedagogía., 173., pp.315-338; Gil Cantero, F. (1991)., El sentido de los derechos humanos en la teoría y práctica educativa., Madrid., Universidad Complutense (Tesis Doctoral); Altarejos, F. y Naval, C., "Virtualidad formativa de los Derechos Humanos: la educación cívica", en Revista Española de Pedagogía., 211., pp.511-527.

Cfr. artículo 13.1. del Pacto Internacional de Derechos Económicos, Sociales y Culturales de 1966 y Oficina del Alto Comisionado de las Naciones Unidas para los Derechos Humanos (1998)., Decenio de las Naciones Unidas para la educación en la esfera de los derechos humanos, 1995-2004. Lecciones para la vida., Nueva York- Ginebra., Naciones Unidas., p.3.

5 Cfr. Altarejos, F. y Naval, C., Virtualidad formativa de los Derechos Humanos: la educación cívica., op. cit.; Naval, C. (2000)., "Educación y derechos humanos", en Humana Iura., 10., pp.43-59; Gil Cantero, F., Jover, G. y Reyero, D., La enseñanza de los derechos humanos: 30 preguntas, 29 respuestas y 76 actividades., Barcelona., Paidós. 
Concretamente, las Naciones Unidas proclamaron en 1995 el "Decenio de las Naciones Unidas para la educación en la esfera de los derechos humanos, 1995-2004” ${ }^{6}$.

En este trabajo expondremos las propuestas metodológicas para la educación en derechos humanos ofrecidas en los textos de las Naciones Unidas y la UNESCO, haciendo para ello un elenco inicial de los textos e iniciativas internacionales más destacables. No obstante, la educación en derechos humanos no sólo incluye el aspecto metodológico. En una propuesta de educación en derechos humanos -y en cualquier propuesta educativa- conviene considerar algunas cuestiones previas, tales como el concepto de educación y las finalidades que con ella se pretenden alcanzar. Además, saber qué enseñar es clave para después elegir los contenidos más adecuados, de ahí la importancia de plantearse la metodología a emplear y seleccionar estrategias de evaluación acertadas. Esa reflexión también ayudará a perfilar el papel que el profesor desempeñará, sin olvidar que la propuesta elaborada para educar en derechos humanos se practica en un clima institucional, social y político concreto ${ }^{7}$.

Asamblea General (1994)., Decenio de las Naciones Unidas para la educación en la esfera de los derechos humanos, 1995-2004., Resolución aprobada en relación con el informe de la Tercera Comisión (A/49/610/Add.2)., Naciones Unidas. Cfr. Ugarte, C. (2004)., Las Naciones Unidas y la educación en derechos humanos., Pamplona., EUNSA., p.16. La teoría de la educación en los derechos humanos que subyace en los textos e iniciativas de estos organismos internacionales se ha estudiado con más profundidad en las pp.225-324 de esta obra. Ésta es parte de una investigación previa que se enmarca en un proyecto de investigación más amplio sobre educación moral y cívica desarrollado en el Departamento de Educación de la Universidad de Navarra. 


\section{LA EDUCACIÓN EN DERECHOS HUMANOS EN LOS TEXTOS E INICIATIVAS DE NACIONES UNIDAS Y UNESCO}

Para hacernos cargo de cuáles son los textos e iniciativas más destacables en los que se refiere a la educación en derechos humanos en Naciones Unidas y UNESCO, haremos referencia a cuatro aspectos: en primer lugar, la "Carta Internacional de Derechos Humanos de las Naciones Unidas"; después a la educación en derechos humanos en otros textos internacionales promulgados por este organismo internacional; en tercer lugar convendrá apuntar al «Decenio de las Naciones Unidas para la Educación en la Esfera de los Derechos Humanos" que Naciones Unidas ha dedicado de 1995 a 2004; y por fin señalar el tratamiento de la cuestión en los textos de la UNESCO ${ }^{8}$.

Para elaborar este epígrafe, se han consultado fundamentalmente las siguientes compilaciones de textos internacionales. En primer lugar el compendio elaborado por la Oficina del Alto Comisionado de las Naciones Unidas en 1999: El Decenio de las Naciones Unidas para la educación en la esfera de los derechos humanos (1995-2004)., № 2., La educación en la esfera de los derechos humanos y tratados de derechos humanos., Nueva YorkGinebra., Naciones Unidas (HR/PUB/DECADE/1999/1); y también The United Nations Decade for Human Rights Education (1995-2004)., № 3., The Right to Human Rights Education. A compilation of provisions of international and regional instruments dealing with human rights education. New York-Geneva: United Nations (HR/PUB/DECADE/1999/2). También la obra de Fernández, A. y Jenker, S. (1995)., Declaraciones y Convenciones Internacionales sobre el Derecho a la Educación y la Libertad de Enseñanza., Frankfurt/Main., Info-3 Verlag. Finalmente, se acudió a Centre for Human Rights (1994)., Human Rights. A compilation of International Instruments., Volume I (First and Second Part): Universal Instruments., Geneva., United Nations (ST/HR/1/Rev.5-Vol. I/ Part 1 and ST/HR/1/Rev.5-Vol. I/ Part 2). 


\section{La Educación en Derechos Humanos en la Carta Internacional de Derechos Humanos de las Naciones Unidas}

La "Carta de Derechos Humanos de las Naciones Unidas" es el documento marco en el que se inspiran todas las iniciativas y trabajos posteriores de este organismo. La Carta abarca diferentes textos internacionales, entre ellos la "Declaración Universal de Derechos Humanos". Los textos de la Carta que incluyen la educación en derechos humanos en su articulado son:

a) La "Declaración Universal de Derechos Humanos" (1948), en el artículo 26.2.

b) El «Pacto Internacional de Derechos Económicos, Sociales y Culturales" (1966), en su artículo 13.1. y 13.3.

c) El "Pacto Internacional de Derechos Civiles y Políticos" (1966), en el artículo 2.2.

Tras enumerar estos artículos, se mencionan ahora los textos posteriores que han tratado este aspecto de la educación.

\section{La Educación en Derechos Humanos en Otros Textos Internacionales de las Naciones Unidas}

Para localizar el tratamiento de la educación en derechos humanos en otros textos internacionales de Naciones Unidas, se han consultado los tratados, declaraciones, recomendaciones, otros instrumentos de Naciones Unidas y también los trabajos de la Comisión de Derechos Humanos. 


\subsection{La educación en derechos humanos en los tratados, declaraciones, recomendaciones y otros instrumentos de las Naciones Unidas}

Son numerosos los textos internacionales que, de manera más o menos directa, hacen referencia a ella. Entre los textos que contemplan la cuestión de la educación en derechos humanos se extraen aquí los artículos de los que se derivan las directrices propuestas en el ámbito internacional. Éstos son:

1. La "Convención de los Derechos del Niño" (1989), concretamente los artículos 4, 17, 19 y 29. Asimismo se podrían subrayar:

a) La Observación General nº 1. Párrafo 1, del artículo 29 de la "Convención de los Derechos del Niño sobre los propósitos de la educación" (2001): en sus artículos 1-4, 8, 9 y $11-15$

b) Las Directrices generales relativas a la forma y el contenido de los informes que deben presentar los Estados Partes de conformidad con el párrafo 1 (b), del artículo 44 de la Convención; en artículo 22 (CRC/C/58).

2. La "Declaración de las Naciones Unidas para la promoción en los jóvenes de los ideales de paz, respeto mutuo y entendimiento" (1965) en sus principios I-III y VI;

3. La Conferencia Internacional sobre Derechos Humanos (Teherán, 1968).

a) En la Resolución No. XX, trata sobre la educación de los jóvenes en el respeto a los derechos humanos y las libertades fundamentales (artículos 1-6 y 8).

b) Y en la Resolución No. XXV, sobre la difusión de la "Declaración Universal de Derechos Humanos". 
4. La "Declaración y Programa de Acción de Viena" (1993), concretamente en artículos 33, 34 y 80-82;

5. La "Declaración sobre el derecho y la responsabilidad de los individuos, los grupos y los órganos de la sociedad a promover y proteger los derechos humanos y las libertades fundamentales reconocidas universalmente" (1998), en sus artículos 5 y 14-16.

En el marco de Naciones Unidas, dentro de la referencia a la educación en derechos humanos que se hace en otros textos internacionales -además de los tratados, declaraciones y recomendaciones referidos-, a continuación, se presentan los principales textos sobre el derecho a la educación, promulgados en el seno de la Comisión de Derechos Humanos.

\subsection{La educación en derechos humanos en los trabajos de la Comisión de Derechos Humanos (1998-2003)}

Dada la importancia que tienen, se nombran aquí los principales informes -con relación a la educación de los derechos humanos- presentados, en plena vigencia del Decenio, a la Comisión de Derechos Humanos de las Naciones Unidas. Los informes estudiados son los siguientes:

1. El Informe Anual del Experto sobre el derecho a la educación, M. Mehedi, presentado de conformidad con la resolución 1997/7 de la Subcomisión de Prevención de las Discriminaciones y Protección de las Minorías, 1998 (E/CN.4/Sub.2/1998/10), artículos 4 y 29. 
2. El Informe Anual del Experto sobre el derecho a la educación, M. Mehedi, presentado de conformidad con la resolución 1997/7 de la Subcomisión de Prevención de las Discriminaciones y Protección de las Minorías, 1999 (E/CN.4/Sub.2/1999/10), artículos 1, 21, 22, 23, 32, 35, 37, 45, 47,50 y 61 .

3. El Informe Anual de la Relatora Especial sobre el derecho a la educación, Katarina Tomasevski, presentado de conformidad con la resolución 2000/9 de la Comisión de Derechos Humanos, 2001 (E/CN.4/2001/52), artículos 8, 9, 11 y 73.

4. El Informe anual de la Relatora Especial sobre el derecho a la educación, Katarina Tomasevski, presentado de conformidad con la resolución 2001/29 de la Comisión de Derechos Humanos, 2002 (E/CN.4/2002/60), artículos 51, 55, 58 y 68 .

Por su especial relevancia dentro de las iniciativas emprendidas por las Naciones Unidas para la promoción de la educación en derechos humanos, se señala seguidamente y de modo sucinto, el contenido del "Decenio de las Naciones Unidas para la educación en la esfera de los derechos humanos" (1995-2004).

\section{El Decenio de las Naciones Unidas para la Educación en la Esfera de los Derechos Humanos, 1995-2004}

En su Resolución 49/184, aprobada el 21 de diciembre de 1994, la Asamblea General de las Naciones Unidas proclamó que el decenio que se iniciaría el 1 de enero de 1995 sería 
designado con el nombre de "Decenio de las Naciones Unidas para la educación en la esfera de los derechos humanos".

La labor de las Naciones Unidas en el desarrollo del Decenio ha sido constante a lo largo de estos años. Para presentar las líneas generales de acción emprendidas en el marco del Decenio, se ha optado por estudiar cuatro iniciativas que parecen centrales ${ }^{\text {? }}$

1. La publicación del "Decenio de las Naciones Unidas para la educación en la esfera de los derechos humanos, 1995-2004. Lecciones para la vida", que recoge la estructura de las actividades y trabajos a realizar ${ }^{10}$.

2. Los informes anuales de la Asamblea General y la Comisión de Derechos Humanos sobre el Decenio. De su lectura se desprenden las principales acciones acometidas durante los años de vigencia del Decenio ${ }^{11}$.

9 Si se quiere consultar el conjunto de acciones emprendidas en el marco del Decenio, se puede acudir a la web del Alto Comisionado de las Naciones Unidas para los Derechos Humanos, en: [http://www.unhchr.ch/spanish/hchr_un_sp.htm]. Además del contenido del Decenio, se presentan distintos materiales y actividades, así como una base de datos sobre educación en derechos humanos.

${ }^{10}$ La referencia completa de esta obra es: Oficina del Alto Comisionado de las Naciones Unidas para los Derechos Humanos (1998), El Decenio de las Naciones Unidas para la educación en la esfera de los derechos humanos 1995-2004. Lecciones para la vida., Nueva York-Ginebra., Naciones Unidas (HR/PUB/DECADE/1998/1).

${ }^{11}$ Los informes estudiados han sido: Asamblea General. Decenio de las Naciones Unidas para la educación en la esfera de los derechos humanos, 1995-2004, y actividades de información pública en la esfera de los derechos humanos., Naciones Unidas., 1998 (A/53/313), 1999 (A/54/399) y 2001 (A/56/271). Comisión de Derechos Humanos., Aplicación del Plan de Acción para el Decenio de las Naciones Unidas para la educación en la esfera de los derechos humanos (1995-2004)., Naciones Unidas., 1999 (E/CN.4/1999/87), 2000 (E/CN.4/2000/93), 2002 (E/CN.4/2002/104) y 2003 (E/CN.4/2003/100). 
3. El «Informe de la Alta Comisionada de las Naciones Unidas para los Derechos Humanos sobre la evaluación de mitad de período de los progresos hechos para alcanzar los objetivos del "Decenio de las Naciones Unidas para la Educación en la esfera de los derechos humanos (19952004)"”. Este informe ofrece tanto los logros como las dificultades encontradas en los cinco primeros años del Decenio. A la luz de los resultados se proponen algunas recomendaciones ${ }^{12}$.

4. El "Informe final sobre el Decenio de las Naciones Unidas para la educación en la esfera de los derechos humanos". Es una medida que se realizará en el futuro para efectuar un balance de los logros y resultados obtenidos al finalizar el Decenio ${ }^{13}$.

Además de las acciones emprendidas en el marco del Decenio, parece obligado hacer una breve referencia a los principales textos de la UNESCO que contemplan la educación de los derechos humanos, y por tanto, también el aspecto metodológico.

2 La nota bibliográfica completa es: Asamblea General (2000)., El Decenio de las Naciones Unidas para la educación en la esfera de los derechos humanos (1995-2004)., Informe de la Alta Comisionada de las Naciones Unidas para los Derechos Humanos sobre la evaluación de mitad de período de los programas hechos para alcanzar los objetivos del Decenio de las Naciones Unidas para la educación en la esfera de los derechos humanos (1995-2004)., Ginebra., Naciones Unidas (A/55/360).

${ }_{13}$ La referencia a este informe, así como las cuestiones que éste procurará incluir cuando se elabore, pueden encontrarse en el apartado X (p.33) del documento anteriormente citado: El Decenio de las Naciones Unidas para la educación en la esfera de los derechos humanos 1995-2004. Lecciones para la vida. 


\section{La Educación en Derechos Humanos en la UNESCO}

Como es sabido, la agencia especializada en educación de las Naciones Unidas es la Organización de las Naciones Unidas para la Educación, la Ciencia y la Cultura (UNESCO). La preocupación de la UNESCO por la educación en derechos humanos es constante. Son numerosas las acciones emprendidas desde este organismo internacional ${ }^{14}$, así como son ingentes las publicaciones sobre este tema. Sin embargo, por razones de espacio, sólo se mencionarán los principales textos que este organismo ha promulgado con relación a la educación en derechos humanos. Éstos son ${ }^{15}$ :

1. La «Recomendación sobre la educación para la comprensión, la cooperación y la paz internacionales, y la educación relativa a los derechos humanos y las libertades fundamentales", de 1974.

${ }_{14}$ Para conocer estas iniciativas se puede acudir a Soler Roca, M. (1988)., "Derechos humanos y educación", en Cuadernos de Pedagogía., 164., pp.815; Levin, L. (1998)., Derechos humanos: preguntas y respuestas., París., UNESCO; Tuvilla Rayo, J. (1998)., La educación en derechos humanos: hacia una perspectiva global., Bilbao., Desclée de Brouwer., Centre for Human Rights (1994)., United Nations action in the field of human rights., Geneva., United Nations (reimpreso en 2001).

${ }_{15}$ Estas acciones de la UNESCO, así como de otras que guardan relación con la educación en derechos humanos, pueden hallarse en la compilación elaborada por la Oficina del Alto Comisionado de las Naciones Unidas para los Derechos Humanos, The United Nations Decade for Human Rights Education (1995-2004)., № 3., The Right to Human Rights Education. A compilation of provisions of international and regional instruments dealing with human rights education., op. cit. Además, el contenido resumido de las cuatro últimas iniciativas mencionadas se puede encontrar en Symonides, J. y Volodin, V. (1995)., "Education for human rights and democracy in the new international context", en VV. AA., Education for human rights and citizenship in Central and Eastern Europe., Praha., Human Rights Centre of EIC of Charles University., pp.28-49. 
2. El Congreso Internacional sobre la enseñanza de los derechos humanos, celebrado en Viena en 1978.

3. La "Recomendación de Malta sobre la enseñanza, documentación e información sobre los derechos humanos", de 1987.

4. El Congreso Internacional sobre Educación para los Derechos Humanos y la Democracia y el Plan de Acción Mundial de la UNESCO para la Educación en Derechos Humanos y la Democracia, efectuado en Montreal, $1993^{16}$.

5. La $44^{\underline{a}}$ reunión de la Conferencia Internacional de Educación, 1994.

Una vez apuntada aquí, de modo sintético, la recopilación de textos y actividades de las Naciones Unidas y la UNESCO que incluyen la educación en derechos humanos en su articulado, se presentan a continuación las líneas metodológicas por las que apuestan estos organismos en referencia a este aspecto de la educación. Las propuestas internacionales se complementarán con algunas precisiones a fin de aportar una visión lo más completa posible de este aspecto didáctico.

${ }^{16}$ El contenido de esta declaración es recogido y comentado por Sánchez Férriz, A. y Jimena, L. (1998)., La enseñanza de los derechos humanos., Barcelona., Ariel., pp.117-128. 


\section{METODOLOGÍA DE LA EDUCACIÓN EN DERECHOS HUMANOS EN LOS TEXTOS DE LAS NACIONES UNIDAS Y LA UNESCO}

Entre todos los textos e iniciativas emprendidas por las Naciones Unidas y la UNESCO que se han mencionado, se dedican muchos artículos al cómo enseñar los derechos humanos ${ }^{17}$. Estos artículos incluyen aspectos metodológicos tan diversos que, para recogerlos de un modo sistemático, se presentan aquí agrupados en categorías ${ }^{18}$ :

a) Marco internacional y estatal.

b) Marco pedagógico.

17 La metodología de la educación en derechos humanos ha sido estudiada por numerosos autores en los últimos años. Se presentan a continuación algunas obras que parecen centrales para poseer una visión amplia que complete y enriquezca este marco internacional: UNESCO (1969)., Algunas sugerencias sobre la enseñanza acerca de los derechos humanos., París., UNESCO; Centro de Derechos Humanos (1989): ABC., La Enseñanza de los Derechos Humanos. Ejercicios prácticos para escuelas primarias y secundarias., Nueva York., Naciones Unidas; Gil Cantero, F. (1991)., El sentido de los derechos humanos en la teoría y práctica educativa., Madrid., Universidad Complutense (Tesis Doctoral) y "La enseñanza de los derechos humanos", en Revista Española de Pedagogía., 190., pp.535-561; Pérez Serrano, G. (1997)., Cómo educar para la democracia. Estrategias educativas., Madrid., Editorial Popular; IDDH-UNESCO (1998)., Manual de Educación en Derechos Humanos. Niveles Primario y Secundario., San José de Costa Rica., IDDHUNESCO; Naval, C. (1998)., "Educación para la ciudadanía", en VV. AA., Filosofía de la educación hoy., Temas., Madrid., Dykinson., pp.355-376; UNESCO (1998)., All human beings... Manual for human rights education., Paris., UNESCO Publishing; Tuvilla, J., La educación en derechos humanos., op. cit..; Zurbano, J. L. (1998)., Bases de una educación para la paz y la convivencia., Pamplona., Departamento de Educación y Cultura., Gobierno de Navarra; Jares, X. R. (1999)., Educación y derechos humanos., Madrid., Editorial Popular; Betancort, I. (2001)., La formación en los valores de los derechos humanos en la educación primaria, una utopía esperanzadora., Madrid., UNED (Tesis Doctoral); Medina, R. (2002)., La formación en los valores de los derechos humanos, fundamento de la convivencia y la paz., Madrid., Real Academia de Doctores.

${ }_{18}$ Para la elaboración de este apartado se ha acudido principalmente al estudio previo realizado por: Ugarte, C., op. cit., pp.316-320. 
c) Ámbito para la educación de los derechos humanos.

d) Valor formativo de la participación para la educación de los derechos humanos.

e) Actividades, recursos y materiales.

a) Con referencia al marco internacional y estatal se señala la conveniencia de:

- Integrar la educación en materia de derechos humanos en las políticas educativas. Éstas, a su vez, habrían de estar asentadas en la dignidad de la persona y en el respeto al pleno desarrollo de sus potencialidades.

- Asignar recursos a programas destinados a fortalecer la legislación, instituciones e infraestructuras nacionales que defiendan la enseñanza y la educación en materia de derechos humanos.

Estas medidas se presentan como muy acertadas, ya que para que la educación en derechos humanos se realice es necesario establecer condiciones políticas, legales y sociales convenientes y todo tipo de medidas institucionales y estructurales. Pero una vez que esto está garantizado, cada persona habrá de asumir su responsabilidad de ejercitar los deberes asociados a todo derecho humano.

b) Respecto al marco pedagógico se aboga por:

- Un planteamiento holístico en educación.

- Desarrollar la dimensión internacional y la perspectiva global de la educación en todos los niveles.

- Un proceso educativo, una organización y unos métodos democráticos en sí mismos.

- Una metodología multidisciplinar.

- Un enfoque metodológico centrado en la experiencia. 
- Unos métodos activos y dinámicos basados en la participación.

- Potenciar la figura del alumno como auténtico agente de su aprendizaje y de su educación.

c) En cuanto al entorno requerido para la educación de los derechos humanos, se mencionan dos ámbitos:

c.1. Ámbito escolar, donde se apunta a:

- Integrar la educación en materia de derechos humanos no sólo en las actividades escolares sino en todo el proceso educativo.

- Que la enseñanza de los derechos humanos no sea una carga del plan de estudios, sino una forma de ampliar las materias. Además de este tratamiento transversal, los derechos humanos también pueden enseñarse como disciplina autónoma -sobre todo en secundaria- en áreas tales como Filosofía, Ciencia Política, Derecho y Teología.

\section{c.2. Ámbito extraescolar, se atiende a que:}

- La educación en derechos humanos esté presente dentro y fuera de los programas de enseñanza (por ejemplo en la familia, las asociaciones y los programas de educación permanente).

d) Con relación al valor formativo de la participación para la educación de los derechos humanos se destaca la idoneidad de fomentar:

- La asociación y concertación entre los distintos actores. Esta educación es responsabilidad compartida de todos los miembros de la comunidad educativa: autoridades públicas, educadores, educandos y familias. 
- La interacción y cooperación de todos los participantes, incluidos los miembros de la sociedad civil.

- La participación de niños, padres y maestros en las decisiones relativas a la educación (siempre según su edad, capacidad y preparación).

- La participación escolar del niño; por ejemplo, mediante la creación de comunidades escolares y consejos de alumnos; la ayuda y asesoramiento entre compañeros; la intervención de los niños en proyectos ambientales locales, regionales o mundiales.

Todas estas tareas siempre contemplarán el sentido de responsabilidad que poseen los alumnos, ya que según su edad estas propuestas acaso puedan resultar poco acertadas. Su responsabilidad se irá estimulando al requerir su colaboración en diferentes tareas según la edad y preparación les permitan.

e) Por último, respecto a actividades, recursos y materiales:

- Las actividades que se emprendan habrán de ser relevantes en la vida diaria de los educandos y por medio de ellas se procurará iniciar un diálogo.

- Fomentar actividades idóneas para educar en derechos humanos: la representación de papeles y los debates.

- Trabajo en grupo y participación en discusiones libres, en las que se concilien valores distintos por medio del diálogo y del respeto a las diferencias.

- Para suscitar la participación escolar, convendrá desarrollar en la enseñanza la utilización de métodos activos, tareas de grupo, discusión sobre cuestiones morales y enseñanza personalizada.

- Implicar a los estudiantes en la organización de los estudios y de la escuela a la que asisten. 
- Utilización de materiales audiovisuales y de medios informativos múltiples. Las tecnologías de la información y las comunicaciones son un importante medio para la difusión de los derechos humanos.

- Empleo de los medios de comunicación.

- Creación de redes docentes.

- Difusión de ideas y materiales innovadores e intercambio de información con países.

- Alentar las investigaciones y la elaboración de material didáctico.

- Eliminar todas las formas de discriminación racial que aparezcan en los libros de texto. Revisión de los materiales escolares para eliminar los estereotipos.

- Métodos didácticos que abarquen conocimientos prácticos, análisis críticos y el desarrollo de aptitudes para promover los derechos humanos.

- Los cursos y materiales de formación habrán de emplearse con flexibilidad, deben ser adaptables a las particularidades de una gama diversa de públicos posibles.

- Acceso a la información y al material procedente de diversas fuentes nacionales e internacionales.

- Difusión mundial de la "Declaración Universal" en la máxima cantidad de idiomas posible y en otras formas apropiadas a los distintos niveles de alfabetización; para los discapacitados es un recurso de gran utilidad para educar en derechos humanos.

Tras esta exposición de las principales líneas metodológicas propuestas por los organismos internacionales se presentan unas reflexiones finales con las que cerraremos el artículo. 


\section{REFLEXIONES FINALES}

1. En los textos de las Naciones Unidas y la UNESCO se otorga especial relevancia al valor formativo de la transmisión de la información así como de la difusión de los textos internacionales. Sin embargo, conocer estos textos no es todo; el conocimiento sin más no es suficiente para educar en derechos humanos, ya que tener información sobre los derechos humanos no significa haberlos hecho propios, que es de lo que se trata al educar en derechos humanos. El conocimiento de los derechos incluidos en la Declaración, no es suficiente para lograr el compromiso con el ejercicio de estos derechos y sus deberes que es lo que se pretende lograr con esta dimensión de la educación.

2. Las propuestas metodológicas de las Naciones Unidas y la UNESCO para enseñar los derechos humanos, abogan principalmente por el empleo de métodos dialógicos y discursivos. Estos métodos son adecuados, pero es necesario complementarlos con otros que incluyan una participación más activa y directa -en la práctica- de quienes se están educando.

Conseguir que los alumnos adopten posturas respetuosas con los derechos humanos en actividades de discusión, simulación y debate en el aula es relativamente sencillo. Sin embargo, que los alumnos lleguen a orientar su vida con los valores de los derechos humanos y se comprometan a humanizar todas sus acciones, no se consigue más que con la práctica de aquellos temas que se discuten en situaciones reales. Además de la disposición al diálogo, serán muy apropiadas otras metodologías tales como fomentar la participación de los alumnos en la dirección y organización de actividades de 
aprendizaje, la prestación de servicios sociales y suscitar el interés del educando en cuestiones cívicas ${ }^{19}$.

Al mismo tiempo, no habrá que olvidar que esta práctica ha de apoyarse sobre un conocimiento y reflexión sobre los derechos humanos que permita adquirir criterios razonados acerca de la idoneidad de vivir los valores de estos derechos.

3. En los textos internacionales se considera que esta educación ha de impartirse en todos los niveles educativos de un modo interdisciplinar. La educación y enseñanza en estos derechos habitualmente se considera como un contenido transversal que ha de impregnar el currículo escolar. Es cierto que la transversalidad se presenta como un medio adecuado para incardinar esta educación en el currículum. Sin embargo, la experiencia demuestra que las áreas transversales no son abordadas como se debiera. Ante esta situación, habría que buscar otros cauces alternativos o complementarios. Una posibilidad podría ser incluir la educación en derechos humanos en una asignatura específica. La existencia de una asignatura no impediría el tratamiento transversal de los derechos humanos en las demás materias. Otra alternativa sería constituir en la escuela un departamento de derechos humanos que coordinase y procurase el desarrollo y aplicación transversal de los derechos en el currículo escolar ${ }^{20}$. Todas ellas son soluciones a experimentar para aplicar la más acertada en cada momento y lugar.

19 Cfr. Gil Cantero, F., El sentido de los derechos humanos en la teoría y práctica educativa., op. cit., pp.206-228; y Medina, R., La formación en los valores de los derechos humanos, fundamento de la convivencia y la paz., op. cit., pp.76-78.

${ }^{20}$ Sobre la idea del departamento de derechos humanos, véase Zurbano, J. L., Bases de una educación para la paz y la convivencia., op. cit., pp.167-169. 


\section{REFERENCIAS BIBLIOGRÁFICAS}

ALTAREJOS, F. y NAVAL, C. (1998)., "Virtualidad formativa de los Derechos Humanos: la educación cívica", en Revista Española de Pedagogía (211)., pp.511-527.

ÁLVAREZ VITA, J. (1998)., "De la Declaración Universal de Derechos Humanos a la globalización: medio siglo de camino (1948-1998)", en Política Internacional., octubre-diciembre., pp. 53-81.

ASAMBlea GEnERAL., Decenio de las Naciones Unidas para la educación en la esfera de los derechos humanos, 1995-2004., y actividades de información pública en la esfera de los derechos humanos., Ginebra., Naciones Unidas., 1998 (A/53/313), 1999 (A/54/399) y 2001 (A/56/271).

ASAMBlea General (2000),, Decenio de las Naciones Unidas para la educación en la esfera de los derechos humanos (1995-2004). Informe de la Alta Comisionada de las Naciones Unidas para los Derechos Humanos sobre la evaluación de mitad de período de los programas hechos para alcanzar los objetivos del Decenio de las Naciones Unidas para la educación en la esfera de los derechos humanos (1995-2004)., Ginebra., Naciones Unidas (A/55/360).

ASAMBlea General (1994),, Decenio de las Naciones Unidas para la educación en la esfera de los derechos humanos, 1995-2004., Resolución aprobada en relación con el informe de la Tercera Comisión., Ginebra., Naciones Unidas (A/49/610/Add.2).

BETANCORT, I. (2001), La formación en los valores de los derechos humanos en la educación primaria, una utopía esperanzadora., Madrid., UNED (Tesis Doctoral). 
CENTRO DE DERECHOS HUMANOS: $A B C$ (1989)., La Enseñanza de los Derechos Humanos. Ejercicios prácticos para escuelas primarias y secundarias., Nueva York., Naciones Unidas.

CENTRE FOR HUMAN RIGHTS (1994)., "Human Rights. A compilation of International Instruments"., Volume I., Universal Instruments (First and Second Part)., Geneva., United Nations (ST/HR/1/Rev.5-Vol. I/ Part 1 and ST/HR/1/Rev.5-Vol. I/ Part 2).

CENTRE FOR HUMAN RIGHTS (1994)., United Nations action in the field of human rights., Geneva., United Nations (reimpreso en 2001).

COMISIÓN DE DERECHOS HUMANOS. Aplicación del Plan de Acción para el Decenio de las Naciones Unidas para la educación en la esfera de los derechos humanos (19952004)., Naciones Unidas., 1999 (E/CN.4/1999/87), 2000 (E/CN.4/2000/93), $2002 \quad$ (E/CN.4/2002/104), 2003 (E/CN.4/2003/100).

COMISIÓN DE DERECHOS HUMANOS (2002), Los Derechos Económicos, Sociales y Culturales., Informe Anual de la Relatora Especial sobre el derecho a la educación, Katarina Tomasevski, presentado por conformidad con la resolución 2001/29 de la Comisión de Derechos Humanos., Ginebra., Naciones Unidas (E/ CN.4/2002/60).

COMISIÓN DE DERECHOS HUMANOS (2001),, Derechos Económicos, Sociales y Culturales., Informe Anual de la Relatora Especial sobre el derecho a la educación Katarina Tomasevski, presentado por conformidad con la resolución 2000/9 de la Comisión de Derechos Humanos., Ginebra., Naciones Unidas (E/ CN.4/2001/52). 
COMISIÓN DE DERECHOS HUMANOS (1999)., Ejercicio de los Derechos Económicos, Sociales y Culturales. El ejercicio del derecho a la educación, inclusive la educación en materia de derechos humanos., Informe Anual del Experto sobre el derecho a la educación, M. Mehedi, presentado de conformidad con la resolución 1997/7 de la Subcomisión de Prevención de las Discriminaciones y Protección de las Minorías., Ginebra., Naciones Unidas (E/CN.4/Sub.2/1999/10).

COMISIÓN DE DERECHOS HUMANOS (1998)., Ejercicio de los Derechos Económicos, Sociales y Culturales., Informe Anual del Experto sobre el derecho a la educación, M. Mehedi, presentado de conformidad con la resolución 1997/7 de la Subcomisión de Prevención de las Discriminaciones y Protección de las Minorías., Ginebra., Naciones Unidas (E/CN.4/Sub.2/1998/10).

DELORS, J. (1996)., La educación encierra un tesoro., Informe a la UNESCO de la Comisión Internacional sobre la educación para el siglo XXI., Madrid., Santillana-Unesco. FERNÁNDEZ, A. y JENKNER, S. (1995)., Declaraciones y Convenciones Internacionales sobre el Derecho a la Educación y la Libertad de Enseñanza., Frankfurt/ Main., Info-3 Verlag.

FERNÁNDEZ, A. (ed.) (2000), Hacia una cultura de los derechos humanos: un manual alternativo de los derechos fundamentales y del derecho a la educación., Ginebra., Universidad de verano de derechos humanos y del derecho a la educación.

GIL CANTERO, F.; JOVER, G.; REYERO, D. (2001)., La enseñanza de los derechos humanos: 30 preguntas, 29 respuestas y 76 actividades., Barcelona., Paidós. 
GIL CANTERO, F. (1991),, El sentido de los derechos humanos en la teoría y práctica educativa., Madrid., Universidad Complutense (Tesis Doctoral).

GIL CANTERO, F. (1991)., "La enseñanza de los derechos humanos", en Revista Española de Pedagogía., 190., pp.535561.

IDDH-UNESCO (1998)., Manual de Educación en Derechos Humanos. Niveles Primario y Secundario., San José de Costa Rica., IDDH-UNESCO.

JARES, X. R. (1999)., Educación y derechos humanos., Madrid., Editorial Popular.

Levin, L. (1998), Derechos humanos: preguntas y respuestas., París., Bakeaz-Ediciones UNESCO.

LYNCH, J.; MODGIL, C.; MODGIL, S. (eds.) (1992)., Human Rights, Education and Global Responsibilities, Cultural Diversity at schools., Volume 4., Washington D.C., The Falmer Press.

MARTÍNEZ, M. y NOGUERA, E. (1998)., "La Declaración Universal de Derechos Humanos: compromisos y deberes", en Revista Española de Pedagogía., 211., pp.483-510.

MEDINA, R. (2002)., La formación en los valores de los derechos humanos, fundamento de la convivencia y la paz., Madrid., Real Academia de Doctores.

MEDINA, R. (1998)., "Los derechos humanos y la educación en los valores de una ciudadanía universal", en Revista Española de Pedagogía., 211., pp.529-559.

MEDINA, R. (1986)., «Educación moral y comportamiento cívicopolítico", en Revista Española de Pedagogía., 173., pp.315-338. MEYER-BISCH, P. (1995)., Cultura democrática: un desafío para las escuelas., París., UNESCO. 
MEYER-BISCH, P. (1998)., Lógicas del derecho a la educación dentro de los derechos culturales., Comité de Derechos Económicos, Sociales y Culturales., Ginebra., Naciones Unidas.

NAVAL, C. (1998)., "Educación para la ciudadanía”, en V.V.A.A., Filosofia de la educación hoy., Temas., Madrid., Dykinson., pp.355-376.

NAVAL, C. (2000)., "Educación y derechos humanos", en Humana Iura., 10., pp.43-59.

OFICINA DEL ALTO COMISIONADO DE LAS NACIONES UNIDAS (1999)., El Decenio de las Naciones Unidas para la educación en la esfera de los derechos humanos (1995-2004)., № 2., La educación en la esfera de los derechos humanos y tratados de derechos humanos., Nueva YorkGinebra., Naciones Unidas (HR/PUB/DECADE/1999/1).

OFFICE OF THE UNITED NATIONS HIGH COMMISSIONER FOR HUMAN RIGHTS (1999)., The United Nations Decade for Human Rights Education (1995-2004)., № 3., The Right to Human Rights Education. A compilation of provisions of international and regional instruments dealing with human rights education., New York-Geneva., United Nations (HR/PUB/DECADE/1999/2).

OFICINA DEL ALTO COMISIONADO DE LAS NACIONES UNIDAS PARA LOS DERECHOS HUMANOS (1998), Decenio de las Naciones Unidas para la educación en la esfera de los derechos humanos, 1995-2004. Lecciones para la vida., Nueva York-Ginebra., Naciones Unidas. PÉREZ SERRANO, G. (1997)., Cómo educar para la democracia. Estrategias educativas., Madrid., Editorial Popular. SÁNCHEZ FERRIZ, R.; JIMENA, L. (1995)., La enseñanza de los derechos humanos., Barcelona., Ariel. 
SYMONIDES, J. y VOLODIN, V. (1995)., "Education for human rights and democracy in the new international context", en VV. AA., Education for human rights and citizenship in Central and Eastern Europe., Praha., Human Rights Centre of EIC of Charles University., pp.28-49.

SOLER ROCA, M. (1988)., "Derechos humanos y educación", en Cuadernos de Pedagogía., 164., pp.8-15.

TUVILla RAYO, J. (1998)., La educación en derechos humanos: hacia una perspectiva global., Bilbao., Desclée de Brouwer.

UGARTE, C. (2004)., Las Naciones Unidas y la educación en derechos humanos., Pamplona., Eunsa.

UNESCO (1969)., Algunas sugerencias sobre la enseñanza acerca de los derechos humanos., París., UNESCO.

UNESCO (1998)., All human beings... Manual for human rights education., Paris., UNESCO Publishing.

UNITED NATIONS (1999)., The right to human rights education. A compilation of provisions of international and regional instruments dealing with human rights education., New York-Geneva., Office of the High Commissioner for Human Rights.

ZURBANO, J. L. (1998)., Bases de una educación para la paz y la convivencia., Pamplona., Departamento de Educación y Cultura., Gobierno de Navarra. 